\title{
Karel Winkelbauer
}

Моменты для сумм случайного числа случайных слагаемых

Czechoslovak Mathematical Journal, Vol. 3 (1953), No. 1, 93-108

Persistent URL: http://dml.cz/dmlcz/100073

\section{Terms of use:}

(C) Institute of Mathematics AS CR, 1953

Institute of Mathematics of the Czech Academy of Sciences provides access to digitized documents strictly for personal use. Each copy of any part of this document must contain these Terms of use.

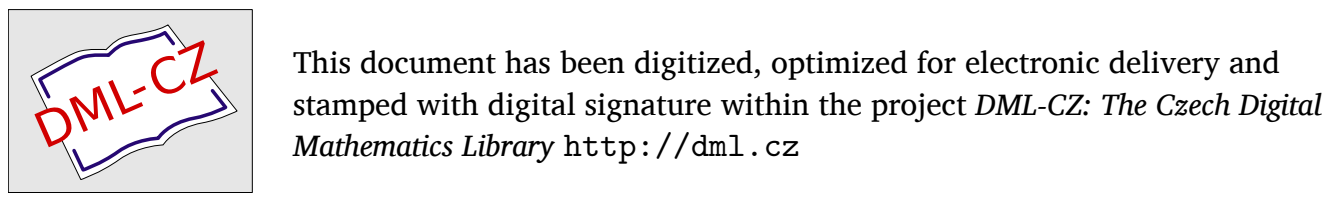




\section{МОМЕНТЫ ДЛЯ СУММ СЛУЧАЙНОГО ЧИСЛА СЛУЧАЙНЫХ СЛАГАЕМЫХ}

КАРЕЛ ВИНКЕЛЬБАУЭР (Karel Winkelbauer), Прага.

(Поступило в редакцию 12/VI 1952 г.)

В настоящей статье приведены некоторые результаты, касающиеся моментов для сумм

$$
z_{n}=x_{1}+x_{2}+\ldots+x_{n}
$$

$n$ первых случайных величин из бесконечной последовательности

$$
x_{1}, x_{2}, \ldots, x_{i}, \ldots,
$$

где само число слагаемых $n$ есть случайная величина. Эти результаты являются в некотором смысле обобщением известных теорем, которые доказали Вальд [3], Блеквель [2], Вольфовиц [4] и Колмогоров-Прохоров [1] (цифры в скобках означают ссылки на список литературы, приведенный в конце статьи).

Статья состоит из пяти частей. Первая содержит некоторое обобщение условий, при которых имеет место формула Вальда

$$
\mathbf{E} z_{n}=a \mathbf{E} n
$$

(см. [1], теорема 1), вторая обобщает в некотором смысле теорему 4 статьи [1] (см. [1] и [5]) и отсюда выведена в третьей части об. щая теорема о моментах более высокого порядка величины $z_{n}$. Четвертая часть содержит лемму вспомогательного характера и в пятой части приведены теоремы о моментах более высокого порядка в случае, когда величины $x_{i}$ взаимно независимы. Последняя теорема, как легко убеждаемся, заключает все результаты Вольфовица в этом паправлении, выведенные в егс статье [4].

Заметим, что во всей статье математические ожидания случайных величин $x$ обозначены через $\mathbf{E}(x)$ и понимаются в смысле абстрактного интеграла Лебега по множеству элементарных событий. Вероятность события, состоящего в том, что имеет место соотношение $S$, обозначена через $\mathbf{P}\{S\}$.

\section{1. Момент первого порядка.}

Во всем дальнейшем $n$ будет обозначать случайную величину, способпую принимать лишь целые неотрицательные значения $i=0,1,2, \ldots$ 
Обозначим через $p_{i}$ вероятность события, состоящего в том, что $n=i$, т. е.

$$
p_{i}=\mathbf{P}\{n=i\} \text {. }
$$

Положим, кроме того,

$$
P_{i}=\mathbf{P}\{n \geqq i\}=\sum_{j=i}^{\infty} p_{j} .
$$

Не нарушая общности, мы ограничимся в настоящей статье случаем, когда $P_{i}>0$ для всех $i$.

Пусть дана последовательность случайных величин $\left\{x_{i}\right\}(i=1,2,3, \ldots)$. Определим случайные величины $z_{i}(i=0,1,2, \ldots)$ как суммы

$$
z_{i}=\sum_{j=1}^{i} x_{j} ;
$$

пс определению пустой суммы $z_{0}=0$. Положим $z_{n}=z_{i}$ для $n=i(i=$ $=0,1,2, \ldots)$. Случайная величина $z_{n}$, следовательно, будет обозначать сумму случайного числа $n$ случайных слагаемых $x_{i}$. Достаточное условие для существования математического ожидания величины $z_{n}$ выражает следующее общее предложение:

Лемма 1. Пусть существуют условные математические ожсидания

$$
a_{i}=\mathbf{E}\left(x_{i} \mid n \geqq i\right),(i=1,2,3, \ldots) .
$$

Положим $c_{i}=\mathbf{E}\left(\left|x_{i}\right| \mid n \geqq i\right)$. Если ряд

$$
\sum_{i=1}^{\infty} P_{i} c_{i}
$$

сходится, то математическое ожсидание случайной величины $z_{n}$ существует. и равно

где $A_{i}=\sum_{j=1}^{i} a_{j}$

$$
\mathbf{E} z_{n}=\sum_{i=1}^{\infty} p_{i} A_{i}
$$

Доказательство этой леммы мы не приводим, так как лемма является лишь более общей формулировкой теоремы, приведенной в статье Колмогорова и Прохорова [1].

Сейчас мы будем исследовать, при каких условиях существует математическое ожидание случайной величины $z_{n}$, если существует математическое ожидание величины $n$. Ответ на этот вопрос дает следующая теорема (которая сформулирована в менее общем виде, хотя ее можно легко сформулировать более обцо):

Теорема 1. Пусть случайная величина $x_{j}$ u собьтие $\{n=i\}$ при $j>i$ независимы. Если существуют математические ожсидания

$$
\mathbf{E} n, a_{i}=\mathbf{E} x_{i},(i=1,2,3, \ldots),
$$


$u$ последовательность $\left\{\mathbf{E}\left|x_{i}\right|\right\}$ ограничена, то математическое охсидание ве.ичинь $z_{n}$ существует и равно

гəe $A_{i}=\sum_{j=1}^{t} a_{j}$

$$
\mathbf{E} z_{n}=\sum_{i=1}^{\infty} p_{i} A_{i}
$$

Доказательство. Положим

$$
c=\sup _{i=1,2,3, \ldots} \mathbf{E}\left|x_{i}\right| .
$$

Из соотношений

$$
c \mathbf{E} n=c \sum_{i=1}^{\infty} p_{i} i=c \sum_{i=1}^{\infty} P_{i} \geqq \sum_{i=1}^{\infty} P_{i} \mathbf{E}\left|x_{i}\right|
$$

вытекает сходимость ряда

$$
\sum_{i=1}^{\infty} P_{i} \mathbf{E}\left|x_{i}\right|
$$

Так как в силу независимости величины $x_{i}$ и события $\{n \geqq i\}$

$$
\mathbf{E}\left(\left|x_{i}\right| / n \geqq i\right)=\mathbf{E}\left|x_{i}\right|, \quad \mathbf{E}\left(x_{i} \mid n \geqq i\right)=\mathbf{E} x_{i},
$$

то утверждение теоремы вытекает из леммы 1.

Очевидным следствием предыдущей теоремы является следующая:

Теорема 2. Пусть случайнал величина $x_{j}$ и событие $\{n=i\}$ при $j>i$ независимы. Если существуют математические ожсидания

$$
\mathbf{E} n, \quad \mathbf{E} x_{i}=a,(i=1,2,3, \ldots),
$$

где а не зависит от $i, u$ последовательность $\left\{\mathbf{E}\left|x_{i}\right|\right\}$ ограничена, то существует математическое ожидание

$$
\mathbf{E} z_{n}=a \mathbf{E} n
$$

\section{2. Момент произведения.}

Пусть теперь дана последовательность $r$-мерных случайных векторов: $\left\{u_{i}\right\}(i=1,2,3, \ldots)$, причем $i$-й случайный вектор имеет $r$ составляющих $u_{i 1}, u_{i 2}, \ldots, u_{i r}(r$ данное натуральное число) или,

$$
u_{i}=\left(u_{i 1}, u_{i 2}, \ldots, u_{i r}\right),
$$

где $u_{i 1}, u_{i 2}, \ldots, u_{i r}$ являются случайными величинами. Определим случайные величины $z_{i k}(k=1,2, \ldots, r ; i=1,2,3, \ldots)$ как суммы

$$
z_{i k}=\sum_{j=1}^{i} u_{j k} .
$$


Положим $z_{n k}=z_{i k}$ при $n=i(k=1,2, \ldots, r)$; случайные величины $z_{n k}$, следовательно, являются суммами случайного числа $n k$-их составляющих векторов $u_{i}$.

Выведем сейчас общую формулу для математического ожидания произведения $\prod_{k=1}^{r} z_{n k}$.

Теорема 3. Пусть для кажсдого натурального числа $i$ выполнено следующее требование: если $v_{1}, v_{2}, \ldots, v_{r}$ натуральные числа такие, что $(a)$ $1 \leqq v_{k} \leqq i(k=1,2, \ldots, r),(б)$ существует по крайней мере один индекс $k$, для которого $v_{k}=i$, то существует условное математическое омсидание

$$
b_{v_{1} \ldots v_{r}}^{(i)}=\mathbf{E}\left(\prod_{k=1}^{r} u_{v_{k} k} \mid n \geqq i\right)
$$

Положим

$$
\beta_{v_{1} \ldots v_{r}}^{(i)}=\mathbf{E}\left(\prod_{k=1}^{r}\left|u_{v_{k} k}\right| \mid n \geqq i\right) .
$$

Нусть при данном $i(i=1,2,3, \ldots)$

$$
\begin{aligned}
& a_{i}=\sum b_{v_{1} \ldots v_{r}}^{(i)}, \\
& c_{i}=\sum \beta_{v_{1} \ldots v_{r}}^{(i)},
\end{aligned}
$$

әде в суммах в правых частях равенств суммируется для всех $v_{1}, v_{2}, \ldots, v_{r}$, для которых выполнены требования (а), (б). Если ряд

$$
\sum_{i=1}^{\infty} P_{i} c_{i}
$$

сходится, то математическое ожсидание произведения $\prod_{k=1}^{r} z_{n k}$ существует и равно

əде $A_{i}=\sum_{j=1}^{i} a_{j}$

$$
\mathbf{E}\left(\prod_{k=1}^{r} z_{n k}\right)=\sum_{i=1}^{\infty} p_{i} A_{i}
$$

Доказательство. Положим

$$
y_{i}=\prod_{k=1}^{r} z_{i k}-\prod_{k=1}^{r} z_{i-1, k} .
$$

Как легко видеть, случайная величина $y_{i}$ является суммой всех $i^{r}-(i-1)^{r}$ произведений вида

$$
u_{v_{1} 1} u_{v_{2} 2} \ldots u_{v_{r} r}
$$

где индексы $v_{1}, v_{2}, \ldots, v_{r}$ выполняют требования (а), (б), приведенные в теореме. Следовательно, условнње математические ожидания

$$
\mathbf{E}\left(y_{i} \mid n \geqq i\right)=a_{i}
$$


существуют, и легко убеждаемся в том, что

$$
\mathbf{E}\left(\left|y_{i}\right| \mid n \geqq i\right) \leqq c_{i},(i=1,2,3, \ldots) .
$$

Так как ряд $\sum_{i=1}^{\infty} P_{i} c_{i}$ сходится, то ряд

$$
\sum_{i=1}^{\infty} P_{i} \mathbf{E}\left(\left|y_{i}\right| \mid n \geqq i\right)
$$

также должен сходиться. Для последовательности $\left\{y_{i}\right\}$, следовательно, выполнены условия леммы 1 , и, значит, существует математическое ожидание

$$
\mathbf{E}\left(\sum_{i=1}^{n} y_{i}\right)=\sum_{i=1}^{\infty} p_{i} A_{i}
$$

Так как

$$
\sum_{i=1}^{n} y_{i}==\prod_{k=1}^{r} z_{n k}
$$

то из предыдущего соотношения вытекает утверждение теоремы, которое и требовалось доказать.

3. Моменты более высоких порядков в общем случае.

Возвратимся теперь к данной последовательности случайных величин $\left\{x_{i}\right\}$ и выведем общую формулу для $r$-го момента величины $z_{n}$ ( $r$ данное натуральное число), причем мы воспользуемся результатом, приведенным в теореме 3 .

Теорема 4. Пусть для кажсдого натурального числа $i$ выполнено следующее требование: если $v_{1}, v_{2}, \ldots, v_{i}$ целье неотрицательнье числа такие, что (a) $0 \leqq v_{k} \leqq r(k=1,2, \ldots, i),(\sigma) \sum_{k=1}^{i} v_{k}=r,(6) v_{i}>0$, то существует условное математическое ожидание

$$
\mathbf{E}\left(x_{1}^{\nu_{1}} x_{2}^{v_{2}} \ldots x_{i}^{v_{i}} \mid n \geqq i\right) .
$$

Для данного $i(i=1,2,3, \ldots)$ поломеим

$$
\begin{aligned}
& a_{i}=\sum \frac{r !}{v_{1} ! \ldots v_{i} !} \mathbf{E}\left(x_{1}^{v_{1}} x_{2}^{v_{2}} \ldots x_{i}^{v_{i}} \mid n \geqq i\right), \\
& c_{i}=\sum \frac{r !}{v_{1} ! \ldots v_{i} !} \mathbf{E}\left(\left|x_{1}\right|^{v_{1}}\left|x_{2}\right|^{\nu_{2}} \ldots\left|x_{i}\right|^{v_{i}} \mid n \geqq i\right),
\end{aligned}
$$

где в суммах в правых частях равенств суммируется ${ }^{\circ}$ ля всех $v_{1}, v_{2}, \ldots, v_{i}$, для которых выполнены требования (а), (б), (в). Если ряд

$$
\sum_{i=1}^{\infty} P_{i} c_{i}
$$


сходится, то существует математическое ожидание

əəе $A_{i}=\sum_{j=1}^{i} a_{j}$

$$
\mathbf{E} z_{n}^{r}=\sum_{i=1}^{\infty} p_{i} A_{i}
$$

Доказательство. Если положить для каждого $i(i=1,2,3, \ldots) u_{i k}=x_{i}$ и значит $z_{i k}=z_{i}, z_{n k}=z_{n}(k=1,2, \ldots, r)$, теорема 4 получается легко из теоремы 3 .

\section{4. Вспомогательная матрица моментов.}

Пусть дана случайная величина $u$, имеющая все моменты до $(r-1)$-го порядка включительно ( $r$ данное натуральное число). Составим квадратную матрицу порядка $r$

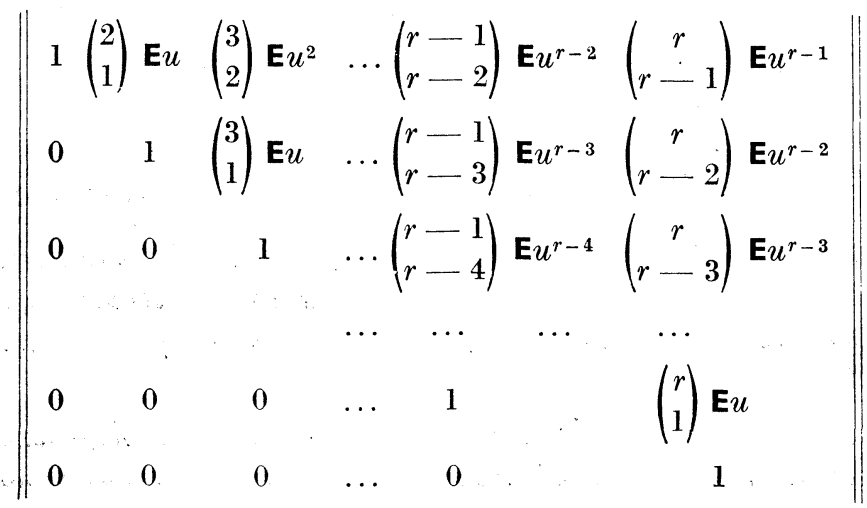

c әлементами под диагональю равными нулю, которую мы обозначим для краткости через $A(u)$. Далее мы обозначим через $A_{i k}(u)$ алгебраическое доголнение элемента, стоящего в $i$-й строке и $k$-м столбце $(i, k=1,2, \ldots, r)$ в определителе матрицы $A(u)$. Выведем сейчас важную лемму, которую мы используем при доказательстве теоремы 5 .

Лемма 2. Если х и у суть две случайћые независимые величины, имеющие все моменты до $(r-1)$-го порядка включительно, то имеют место равенства (при $s=1,2 . \ldots, r)$

$$
\sum_{k=s}^{r}\left(\begin{array}{c}
k \\
k-s
\end{array}\right) \mathbf{E} y^{k-s} A_{r k}(x+y)=A_{r s}(x) .
$$

Доказательство. Обозначим через $B(u)$ квадратную матрицу порядка $r$, имеющую в первом столбце әлементы $A_{r 1}(u), A_{r 2}(u), \ldots, A_{r r}(u)$ и во всех остальных столбцах нули. Тогда мы можем записать систему (при $s=1$, $2, \ldots, r)$ равенств (10) в матричном виде следующим образом: 


$$
A(y) B(x+y)=B(x) .
$$

С. целью доказателсьтва приведенного соотношения (11) выведем теперь следующую формулу:

$$
A(x+y)=A(x) A(y) .
$$

Если обозначим әлементы матрицы; являющейся произведением матриц $A(x)$ и $A(y)$, через $c_{i k}(i, k=1,2, \ldots, r)$, получаем при $i>k: c_{i k}=0$, а при $i \leqq k$, в силу независимости величин $x$ и $y$,

$$
\begin{aligned}
& c_{i k}=\sum_{j=1}^{k}\left(\begin{array}{c}
j \\
j-i
\end{array}\right)\left(\begin{array}{c}
k \\
k-j
\end{array}\right) \mathbf{E} x^{j-i} \mathbf{E} y^{k-j}= \\
& =\left(\begin{array}{c}
k \\
k-i
\end{array}\right) \sum_{j=0}^{k-i}\left(\begin{array}{c}
k-i \\
j
\end{array}\right) \mathbf{E} x^{j} \mathbf{E} y^{k-j-i}= \\
& =\left(\begin{array}{c}
k \\
k-i
\end{array}\right) \mathbf{E}\left[\sum_{j=0}^{k-i}\left(\begin{array}{c}
k-i \\
j
\end{array}\right) x^{j} y^{k-j-i}\right]= \\
& =\left(\begin{array}{c}
k \\
k-i
\end{array}\right) \mathbf{E}(x+y)^{k-i} \text {. }
\end{aligned}
$$

Отсюда следует, что имеет место формула (12).

Если обозначить, как обычно, через $A^{-1}(u)$ матрицу обратную к $A(u)$, мы получаем из формулы (12)

$$
A^{-1}(x+y)=A^{-1}(y) A^{-1}(x) .
$$

Пользуясь формулой (13), легко выводим следующие соотношенйя для $k=1,2, \ldots, r$ :

или, в матричном виде,

$$
A_{r l i}(x+y)=\sum_{j=k}^{r} A_{r j}(x) A_{\jmath k}(y)
$$

$$
B(x+y)=A^{-1}(y) B(x) .
$$

Умножая равенство (14) на матрицу $A(y)$ в соответствующем порядке, мы получаем соотнонение (11), которое и требовалось доказать.

5. Моменты более высоких порядков в случаенезависимости.

Далее при рассмотрении моментов более высокых порядков величины $z_{n}$ мы будем предполагать, что случайные величины $x_{i}$ данной последовательности $\left\{x_{i}\right\}$ взаимно независимы и что случайная величина $n$ удовлетворяет следуюцим требованиям:

1. $\mathbf{P}\{n=0\}=p_{0}=0$;

2. для каждого $i(i=1,2,3, \ldots)$ существует множество $S_{i} i$-мерного евклидового пространства такое, что

$$
\{n=i\}=\left(x_{1}, x_{2}, \ldots, x_{i}\right)^{-1}\left(S_{i}\right),
$$


причем через выражение в правой части (а) обозначено множество всех әлементарных событий, для которых вектор $\left(x_{1}, x_{2}, \ldots, x_{i}\right)$ лежит в множестве $S_{i}$.

Теорема 5. IIусть существует математическое ожидание

$$
\mathbf{E}\left(\sum_{i=1}^{n}\left|x_{i}\right|\right)^{r}
$$

(г данное натуральное число). Поломсим (для $i=1,2,3, \ldots)$

$$
c_{k i}=\mathbf{E}\left(\sum_{j=1}^{i}\left|x_{j}\right|\right)^{r-k}, \quad(k=0,1, \ldots, r) .
$$

Пусть далее существуют математические ожидания

$$
\mathbf{E}\left[c_{k n}\left(\sum_{i=1}^{n}\left|x_{i}\right|\right)^{k}\right]
$$

при $k=1,2, \ldots, r-1$. Положим $($ для $i=1,2,3, \ldots)$

$$
\begin{gathered}
a_{k i}=-A_{r k}\left(z_{i}\right),(k=1,2, \ldots, r-1), \\
a_{0 i}=\sum_{k=1}^{r} \mathbf{E} z_{i}^{k} A_{r k}\left(z_{i}\right),
\end{gathered}
$$

где через $A_{r k}\left(z_{i}\right)$ обозначены соответствующие алгебраические дополнения әлементов в определителе матрищь $A\left(z_{i}\right)$. Тогда существует момент $r$-го порядка случайной величины $z_{n}$ и он равен

$$
\mathbf{E}\left(z_{n}^{r}\right)=\mathbf{E}\left(a_{0 n}\right)+\mathbf{E}\left(a_{1 n} z_{n}\right)+\mathbf{E}\left(a_{2 n} z_{n}^{2}\right)+\ldots+\mathbf{E}\left(a_{r-1}, z_{n} z_{n}^{r-1}\right) .
$$

Доказательство. Легко убедимся в том, что математическое ожидание (15) можно написать в виде ряда

$$
\sum_{i=1}^{\infty} \int_{\{n \geqq i\}}\left\{\sum_{l=1}^{r}\left(\begin{array}{l}
r \\
l
\end{array}\right)\left(\sum_{j=1}^{i-1}\left|x_{j}\right|\right)^{r-l}\left|x_{i}\right|^{l}\right\} \mathrm{d} P .
$$

В силу того, что случайная величина $x_{i}$ независима от суммы $\sum_{j=1}^{i-1}\left|x_{j}\right|$ и, одновременно, и от события $\{n \geqq i\}$, мы можем переписать этот ряд в виде

$$
\sum_{i=1}^{\infty} \sum_{l=1}^{r}\left(\begin{array}{l}
r \\
l
\end{array}\right) \mathbf{E}\left|x_{i}\right|^{l} \int_{\{n \geq i\}}\left(\sum_{j=1}^{i-1}\left|x_{j}\right|\right)^{r-l} \mathrm{~d} P .
$$

Отсюда ясно, что случайные величины $x_{i}$ имеют все моменты до $r$-го порядка включительно и, следовательно, что существуют математические ожидания

$$
c_{k i}=\mathbf{E}\left(\sum_{j=1}^{i}\left|x_{j}\right|\right)^{r-k}
$$

и выражения $a_{k i}$ определены. 
Из соотношений

$$
\begin{aligned}
& \sum_{k=1}^{r}\left(\begin{array}{l}
r \\
k
\end{array}\right) \mathbf{E}\left[c_{k n}\left(\sum_{i=1}^{n}\left|x_{i}\right|\right)^{k}\right]= \\
& =\sum_{k=1}^{r}\left(\begin{array}{l}
r \\
k
\end{array}\right) \sum_{i=1}^{\infty} \int_{\{n=i\}}\left\{c_{k i}\left(\sum_{j=1}^{i}\left|x_{j}\right|\right)^{k}-c_{k, i-1}\left(\sum_{j=1}^{i-1}\left|x_{j}\right|\right)^{k}\right\} \mathrm{d} P \geqq \\
& \geqq \sum_{i=1}^{r}\left(\begin{array}{l}
r \\
k
\end{array}\right) \sum_{i=1}^{\infty} c_{k, i-1} \int_{\{n \geqq i\}}\left|x_{i}\right|^{k} \mathrm{~d} P= \\
& =\sum_{i=1}^{\infty} P_{i} \sum_{k=1}^{r}\left(\begin{array}{l}
r \\
k
\end{array}\right) \mathbf{E}\left(\sum_{j=1}^{i-1}\left|x_{j}\right|\right)^{r-k} \mathbf{E}\left|x_{i}\right|^{k}= \\
& \left.=\sum_{i=1}^{\infty} P_{i}\left\{\mathbf{E}\left(\sum_{j=1}^{i}\left|x_{j}\right|\right)^{r}-\mathbf{E} \sum_{j=1}^{i-1}\left|x_{j}\right|\right)^{r}\right\}= \\
& =\sum_{i=1}^{\infty} P_{i}\left(c_{0 i}-c_{0 . i-1}\right)=\sum_{i=1}^{\infty} p_{i} c_{0 i}=\mathbf{E}\left(c_{0 n}\right)
\end{aligned}
$$

следует, что существует математическое ожидание $\mathbf{E}\left(c_{0 n}\right)$. Покажем, что существуют математические ожидания $\mathbf{E}\left(a_{k n} z_{n}^{k}\right)$ (при $k=0,1, \ldots, r-1$ ). Алгебраическое дополнение $A_{r k}(u)$, как легко видеть, является суммой произведений вида

$$
c_{v_{1} \ldots v_{r-k}}(\mathbf{E} u)^{v_{1}}\left(\mathbf{E} u^{2}\right)^{v_{2}} \ldots\left(\mathbf{E} u^{r-k}\right)^{v_{r-k}},
$$

где $\sum_{s=1}^{r-k} s v_{s}=r-\underset{r-k}{k}$ и $c_{v_{1} \ldots v_{r-k}}$ суть постоянные. В силу неравенств Гельдера имеем при $\sum_{s=1}^{r-k} s v_{s}=r-k$

$$
\left|(\mathbf{E} u)^{v_{1}}\left(\mathbf{E} u^{2}\right)^{v_{2}} \ldots\left(\mathbf{E} u^{r-k}\right)^{v_{r-k}}\right| \leqq \mathbf{E}|u|^{r-k} .
$$

Отсюда следует, что (при $k=0,1, \ldots, r-1)$

$$
\left|\mathbf{E}\left(a_{k n} z_{n}^{k}\right)\right| \leqq N_{k} \mathbf{E}\left[c_{k n}\left(\sum_{i=1}^{n}\left|x_{i}\right|\right)^{k}\right],
$$

где $N_{k}$ постоянно; значит, что $\mathbf{E}\left(a_{k n} z_{n}^{k}\right)$ существуют.

Из существования математического ожидания (15) легко вытекает, что существуют интегралы (при $k=1,2, \ldots, r ; i=1,2,3, \ldots$ )

$$
\int_{\{n \geqq i\}} z_{i}^{k} \mathrm{~d} P, \int_{\{n \geqq i\}} z_{i-1}^{k} \mathrm{~d} P .
$$

Покажем, что имеет место равенство

$$
\int_{\{n \geqq i\}}\left(z_{i}^{r}-z_{i-1}^{r}\right) \mathrm{d} P=\int_{\{n \geqq i\}} \sum_{k=0}^{r-1}\left(a_{k i} z_{i}^{k}-a_{k, i-1} z_{i-1}^{k}\right) \mathrm{d} P
$$

$(i=1,2,3, \ldots)$. Вычисляя интеграл в правой части (18) и пользуясь при 
этом независимостью случайной величины $x_{i}$ от величины $z_{i-1}$ вместе с событием $\{n \geqq i)$, получим выражение

$$
\begin{gathered}
\sum_{k=1}^{r-1} A_{r k}\left(z_{i}\right)\left\{\sum_{j=0}^{k}\left(\begin{array}{l}
k \\
j
\end{array}\right) \mathbf{E} x_{i}^{k-j}\left(P P_{i} \mathbf{E} z_{i-1}^{j}-\int_{\{n \geqq i\}} z_{i-1}^{j} \mathrm{~d} P\right)\right\}- \\
-\sum_{k=1}^{r-1} A_{r k}\left(z_{i-1}\right)\left(P_{i} \mathbf{E} z_{i-1}^{k}-\int_{\{n \geqq i\}} z_{i-1}^{k} \mathrm{~d} P\right)+ \\
+P_{i}\left(\sum_{j=0}^{r}\left(\begin{array}{l}
r \\
j
\end{array}\right) \mathbf{E} x_{i}^{r-j} \mathbf{E} z_{i-1}^{j}-\mathbf{E} z_{i-1}^{r}\right) .
\end{gathered}
$$

Последнее выражение можно записать в виде

$$
\begin{gathered}
\sum_{s=1}^{r-1}\left[\sum_{k=s}^{r-1} A_{r k}\left(z_{i}\right)\left(\begin{array}{c}
k \\
k-s
\end{array}\right) \mathbf{E} x_{i}^{k-s}-A_{r s}\left(z_{i-1}\right)\right] \cdot\left[P_{i} \mathbf{E} z_{i-1}^{s}-\int_{\{n i\}} z_{i-1}^{s} \mathrm{~d} P\right]+ \\
+P_{i} \sum_{s=0}^{r-1}(r-s) \mathbf{E} x_{i}^{r-s} \mathbf{E} z_{i-1}^{s} .
\end{gathered}
$$

Пользуясь леммой 2 , заменяя в (10) $x$ величиной $z_{i-1}$ и $y$ величиной $x_{i}$, имеем (при $s=1,2, \ldots, r)$ :

$$
\sum_{k=s}^{r-1} A_{r k}\left(z_{i}\right)\left(\begin{array}{c}
k \\
k-s
\end{array}\right) \mathbf{E} x_{i}^{k-s}-A_{r s}\left(z_{i-1}\right)=-\left(\begin{array}{c}
r \\
r-s
\end{array}\right) \mathbf{E} x_{i}^{r-s} .
$$

После подстановки (20) в (19), выраженне (19) перейдет в следующее:

$$
\sum_{s=1}^{r-1}(r-s) \mathbf{E} x_{i}^{r-s}\left[\int_{\{n \geqq i\}} z_{i-1}^{s} \mathrm{~d} P-P_{i} \mathbf{E} z_{i-1}^{s}\right]+P_{i} \sum_{s=0}^{r-1}\left(\begin{array}{c}
r \\
r-s
\end{array}\right) \mathbf{E} x_{i}^{r-s} \mathbf{E} z_{i-1}^{s} .
$$

После упрощения это выражение примет вид

$$
\int_{\{n \geqq i\}} \sum_{s=1}^{r}\left(\begin{array}{l}
r \\
s
\end{array}\right) x_{i}^{r-s} z_{i-1}^{s} \mathrm{~d} P,
$$

что есть не что иное, как интеграл

$$
\int_{\{n \geqq i\}}\left(z_{i}^{r}-z_{i-1}^{r}\right) \mathrm{d} P .
$$

Итак, мы доказали равенство (18), что-и требовалось.

Перейдем теперь к доказательству формулы (17). Так как существует математическое ожидание (15), должен ряд

$$
\sum_{j=1}^{\infty} \int_{\{n=j\}}\left(\sum_{i=1}^{j} x_{i}\right)^{r} \mathrm{~d} P
$$

абсолютно сходиться, т. е. существует математическое ожидание $\mathbf{E} z_{n}^{r}$. Абсолютная сходимость последнего ряда гарантирует законность следующих замен порядка суммирования, если мы при этом воспользуемся доказанным равенством (18): 


$$
\begin{aligned}
\mathbf{E} z_{n}^{r} & =\sum_{j=1}^{\infty} \sum_{i=1}^{j} \int_{\{n=j\}}\left(z_{i}^{r}-z_{i-1}^{r}\right) \mathrm{d} P=\sum_{i=1}^{\infty} \sum_{j=i}^{\infty} \int_{\{n=j\}}\left(z_{i}^{r}-z_{i-1}^{r}\right) \mathrm{d} P= \\
& =\sum_{i=1}^{\infty} \int_{\{\geqq i\}}\left(z_{i}^{r}-z_{i-1}^{r}\right) \mathrm{d} P=\sum_{i=1}^{\infty} \int_{\{n \geqq i\}} \sum_{k=0}^{r-1}\left(a_{k i} z_{i}^{k}-a_{k, i-1} z_{i-1}^{k}\right) \mathrm{d} P= \\
& =\sum_{i=1}^{\infty} \sum_{j=i}^{\infty} \sum_{k=0}^{r-1} \int_{\{n=j\}}\left(a_{k i} z_{i}^{k}-a_{k, i-1} z_{i-1}^{k}\right) \mathrm{d} P=\sum_{j=1}^{\infty} \sum_{k=0}^{r-1} \int_{\{n=j\}} a_{k j} z_{j}^{k} \mathrm{~d} P .
\end{aligned}
$$

Так как $\mathbf{E}\left(a_{k n} z_{n}^{k}\right)$ существуют, получаем:

$$
\sum_{j=1}^{\infty} \sum_{k=0}^{r-1} a_{k j} \int_{\{n=j\}} z_{j}^{k} \mathrm{~d} P=\sum_{k=0}^{r-1} \sum_{j=1}^{\infty} a_{k j} \int_{\{n=j\}} z_{j}^{k} \mathrm{~d} P .
$$

Следовательно, соотношение (17) выполнено, чем и заканчивается доказательство теоремы 5.

Замечание. Утверждение теоремы 5 имеет место также в случае, когда существуют математические ожидания

$$
\mathbf{E} x_{i}^{r}, \quad \mathbf{E}\left[c_{k n}\left(\sum_{i=1}^{n}\left|x_{i}\right|\right)^{k}\right], k=0,1,2, \ldots, r-1,
$$

так как из соотношений

$$
\begin{gathered}
\sum_{k=0}^{r-1}\left(\begin{array}{l}
r \\
k
\end{array}\right) \mathbf{E}\left[c_{k n}\left(\sum_{i=1}^{n}\left|x_{i}\right|\right)^{k}\right]=\sum_{i=1}^{\infty} \sum_{k=0}^{r-1}\left(\begin{array}{l}
r \\
k
\end{array}\right) \int_{\{n \geqq i\}}\left\{c_{k i}\left(\sum_{j=1}^{i}\left|x_{j}\right|\right)^{k}-c_{k, i-1}\left(\sum_{j=1}^{i-1}\left|x_{j}\right|\right)^{k}\right\} \mathrm{d} P \geqq \\
\geqq \sum_{i=1}^{\infty} \sum_{k=0}^{r-1}\left(\begin{array}{l}
r \\
k
\end{array}\right) \mathbf{E}\left|x_{i}\right|^{r-k} \int_{\{n \geqq i}\left(\sum_{j=1}^{i-1}\left|x_{j}\right|\right)^{k} \mathrm{~d} P
\end{gathered}
$$

следует, что существует математическое ожидание

$$
\mathbf{E}\left(\sum_{i=1}^{n}\left|x_{i}\right|\right)^{r} .
$$

Очевидным следствием теоремы 5 является следующая:

Теорема 6. Пусть существуют математические ожидания

$$
\mathbf{E}\left[n^{r-k}\left(\sum_{i=1}^{n}\left|x_{i}\right|\right)^{k}\right], k=1,2, \ldots, r,
$$

u последовательность $\left\{\mathbf{E}\left|x_{i}\right|^{r}\right\}$ ограничена. Тогда имеет место формула (17). Если еще $0<\varepsilon \leqq \mathbf{E}\left|x_{i}\right|$ для всех $i$, где $\varepsilon$ некоторое положсительное число, то существует момент $r$-го порядка случайной величины $n$.

Замечание. Из замечания к теореме 5 следует, что утверждение теоремы справедливо также в случае, если существуют математические ожидания

$$
\dot{\mathbf{E}} x_{i}^{r}, \quad \mathbf{E}\left[n^{r-k}\left(\sum_{i=1}^{n}\left|x_{i}\right|\right)^{k}\right], k=0,1,2, \ldots, r-1,
$$

и последовательность $\left\{\mathbf{E}\left|x_{i}\right|^{r}\right\}$ ограничена. 
Теорема 7. Пусть существуют математические ожидания

$$
w_{i}=\mathbf{E} x_{i}, \quad \mathbf{E}\left(\sum_{i=1}^{n}\left|x_{i}-w_{i}\right|\right)^{r}, \quad \mathbf{E}\left[n^{r-k-1}\left(\sum_{i=1}^{n}\left|x_{i}-w_{i}\right|\right)^{k}\right], k=1,2, \ldots, r-2
$$

$(r$ данное натуральное иисло $>1)$. Если $\mathbf{E}\left|x_{i}-w_{i}\right| \geqq \varepsilon, \quad \mathbf{E}\left|x_{i}-w_{i}\right|^{r} \leqq \frac{1}{\varepsilon}$ для всех $i$, аде $\varepsilon$ - некоторое поломсительное число меньше единицы, то существует

$$
\mathbf{E}\left(z_{n}-W_{n}\right)^{r}=\sum_{k=\mathbf{0}}^{r-2} \mathbf{E}\left[b_{k n}\left(z_{n}-W_{n}\right)^{k}\right],
$$

приче.и

$$
\begin{aligned}
W_{i} & =\sum_{j=1}^{i} w_{j}, \\
b_{k i} & =-A_{r k}\left(z_{i}-W_{i}\right), k=1,2, \ldots, r-2, \\
b_{0 i} & =\sum_{k=1}^{r} \mathbf{E}\left(z_{i}-W_{i}\right)^{k} A_{r k}\left(z_{i}-W_{i}\right) .
\end{aligned}
$$

Доказательство. Теорема доказывается подобным же образом как теоремы предыдущие, причем воспользуемся оценкой

$$
\begin{gathered}
\left|\mathbf{E}\left(z_{i}-W_{i}\right)^{k}\right| \leqq \frac{1}{\varepsilon} \sum_{j=0}^{k}(-1)^{j}\left(\begin{array}{l}
k \\
j
\end{array}\right)\left(\begin{array}{l}
i \\
j
\end{array}\right) j !(i-j)^{k-j} \leqq \\
\leqq \text { const } \cdot \frac{1}{\varepsilon} i^{k-1}, i \geqq k, k=2,3, \ldots, r .
\end{gathered}
$$

Замечание. В частном случае $r=3$ достаточно предполагать лишь существование

$$
\mathbf{E}\left(\sum_{i=1}^{n}\left|x_{i}-w_{i}\right|\right)^{3},
$$

так как тогда существует $\mathbf{E}(n)$ и, значит, также $\mathbf{E}\left(b_{0 n}\right)$.

\section{ЛИТЕРАТУРА}

[1] А.Н. Колмогоров - Ю.В.Прохоров: О суммах случайного числа случайных слагаемых, Усп. матем. наук, том 4 (1949), вып. 4 (32), 168-172.

[2] D. Blackwell: On an equation of Wald, Ann. of Math. Stat., vol. 17 (1946), 84-87.

[3] A. Wald: Differentiation under the expectation sign in the fundamental identity of sequential analysis, Ann. of Math. Stat., vol. 17 (1946), 493-497.

[4] $J$. Wolfowitz: The efficiency of sequential estimates and Wald's equation for sequential processes, Ann. of Math. Stat., vol. 18 (1947), 215-230.

[5] J.Seitz - K. Winkelbauer: Заметка к статье Колмогорова и Прохорова ,О суммах случайного числа случайных слагаемых", Чехословацкий математический журнал, 78 (1953), 89—91. 
Summary.

\title{
MOMENTS FOR CUMULATIVE SUMS OF RANDOM VARIABLES
}

\author{
KAREL WINKELBAUER, Prague.
}

(Received June 12, 1952.)

In the preceding article $n$ denotes a random variable which can assume only non-negative integral values $i=0,1,2, \ldots$ The probability that $n=i$ holds is denoted by $p_{i}$ and

$$
P_{i}=\mathbf{P}\{n \geqq i\}=\sum_{j=i}^{\infty} p_{j} .
$$

Without loss of generality we assume that $P_{i}>0$ for all $i$. Further $\left\{x_{i}\right\}_{1}^{\infty}$ is a given sequence of random variables and $z_{i}$ are defined as sums (1). We define the random variable $z_{n}$ by

$$
z_{n}=z_{i} \text { for } n=i \text {. }
$$

Lemma 1. If the conditional expected values (2) exist and the series

$$
\sum_{i=1}^{\infty} P_{i} \mathbf{E}\left(\left|x_{i}\right| / n \geqq i\right)
$$

is convergent, the expected value of the random variable $z_{n}$ exists and is equal to

$$
\mathbf{E} z_{n}=\sum_{i=1}^{\infty} p_{i} A_{i}, \quad A_{i}=\sum_{j=1}^{i} a_{j} .
$$

Theorem 1. Let the random variable $x_{j}$ and the event $\{n=i\}$ for $j>i$ be independent. If the expected values (3) exist and the sequence $\left\{\mathbf{E}\left|x_{i}\right|\right\}$ is bounded, there exists the expected value of the random variable $z_{n}$ and is equal to

$$
\mathbf{E} z_{n}=\sum_{i=1}^{\infty} p_{i} A_{i}, \quad A_{i}=\sum_{j=1}^{i} a_{j} .
$$

Theorem 2. Let the random variable $x_{j}$ and the event $\{n=i\}$ for $j>i$ be independent. If the expected values

$$
\mathbf{E} n, \quad \mathbf{E} x_{i}=a,(i=1,2,3, \ldots),
$$

exist, where a is independent of the subscript $i$, and the sequence $\left\{\mathbf{E}\left|x_{i}\right|\right\}$ is bounded, there exists the expected value of the random variable $z_{n}$ and is equal to

$$
\mathbf{E} z_{n}=a \mathbf{E} n .
$$

Theorem 1 is implied by Lemma 1 and contains Theorem 2 concerning the well-known Wald's identity as a particular case.

If $\left\{u_{i}\right\}_{i=1}^{\infty}$ is a sequence of $r$-dimensional random vectors $u_{i}$ with components $u_{i 1}, u_{i 2}, \ldots, u_{i r}, z_{i k}$ are defined as sums (4), and $z_{n k}$ are defined by 


$$
z_{n k}=z_{i k} \text { for } n=i,(k=1,2, \ldots, r),
$$

then the following theorem holds:

Theorem 3. Suppose that the following condition is fulfilled for each positive tnteger $i$ : whenever $v_{1}, v_{2}, \ldots, v_{r}$ are positive integers such that $(a) 1 \leqq v_{k} \leqq i$ $(k=1,2, \ldots, r),(\mathrm{b}) v_{k}=i$ for at least one $k$, there exists the conditional expecied value (5). Writing

$$
\beta_{\nu_{1} \ldots v_{r}}^{(i)}=\mathbf{E}\left(\prod_{k=1}^{r}\left|u_{v_{k} k}\right| \mid n \geqq i\right)
$$

let

$$
\begin{aligned}
& a_{i}=\sum b_{v_{1} \ldots v_{r}}^{(i)}, \\
& c_{\imath}=\sum \beta_{v_{1} \ldots v_{r}}^{(i)},
\end{aligned}
$$

where the subscripts $v_{1}, v_{2}, \ldots, v_{r}$ run through all the $i^{r}-(i-1)^{r}$ possible values satistying conditions (a), (b). If the series (6) is convergent, there exists the expected value of the product $\prod_{k=1}^{r}\left[z_{n k}\right.$ and is equal to

$$
\mathbf{E}\left(\prod_{k=1}^{r} z_{n k}\right)=\sum_{i=1}^{\infty} p_{i} A_{i}, A_{i}=\sum_{j=1}^{i} a_{j} .
$$

Theorem 3 is a consequence of Lemma 1 and implies the following theorem concerning the given sequence $\left\{x_{i}\right\}$ of the random variables $x_{i}$ :

Theorem 4. Suppose that the following condition is fulfilled for each positive integer $i$ : whenever $v_{1}, v_{2}, \ldots, v_{i}$ are non-negative integers such that $(a) 0 \leqq v_{k} \leqq r$ $(k=1,2, \ldots, i)$, (b) $\sum_{k=1}^{i} v_{k}=r$, (c) $v_{i}>0$, there exists the conditional expected value (7). Let

$$
\begin{aligned}
a_{i} & =\sum \frac{r !}{v_{1} ! \ldots v_{i} !} \mathbf{E}\left(x_{1}^{v_{1}} x_{2}^{v_{2}} \ldots x_{i}^{v_{i}} \mid n \geqq i\right), \\
c_{i} & =\sum \frac{r !}{v_{1} ! \ldots v_{i} !} \mathbf{E}\left(\left|x_{1}\right|^{v_{1}}\left|x_{2}\right|^{v_{2}} \ldots\left|x_{i}\right|^{v_{i}} \mid n \geqq i\right),
\end{aligned}
$$

where the exponents $v_{1}, v_{2}, \ldots, v_{i}$ run through all the $i^{r}-(i-1)^{r}$ possible values satisfying conditions $(a),(b),(c)$. If the series (8) is convergent, then the moment of order $r$ of the random variable $z_{n}$ exists and is equal to

$$
\mathbf{E} z_{n}^{r}=\sum_{i=1}^{\infty} p_{i} A_{i}, A_{i}=\sum_{j=1}^{i} a_{j} .
$$

If $u$ is a random variable and $\mathbf{E} u^{r-1}$ exists ( $r$ is a given positive integer), then $A(u)$ denotes the moment matrix (9) and $A_{i k}(u)$ denotes the cofactor of the element standing in the $i^{\text {th }}$ row and $k^{\text {th }}$ column in the determinant of the matrix $A(u)$. 
Lemma 2. If $x$ and $y$ are two independent random variables and $\mathbf{E} x^{r-1}, \mathbf{E} y^{r-1}$ exist, the the relations (10) hold for $s=1,2, \ldots, r$.

Suppose that the random variables $x_{i}$ of the given sequence $\left\{x_{i}\right\}$ are independent and the random variable $n$ satisfies the following conditions:

1. $\mathbf{P}\{n=0\}=p_{0}=0$;

2. there exists for each $i(i=1,2,3, \ldots)$ a set $S_{i}$ of the $i$-dimensional Euclidean space such that

$$
\{n=i\}=\left(x_{1}, x_{2}, \ldots, x_{i}\right)^{-1}\left(S_{i}\right),
$$

where the expression on the right side of (a) denotes the set of all the elementary events for which the vector $\left(x_{1}, x_{2}, \ldots, x_{i}\right)$ belongs to $S_{\imath}$.

Then the following proposition holds:

Theorem 5. Let the expected value (15) exists ( $r$ is a given positive integer). Putting

$$
\begin{aligned}
& c_{k i}=\mathbf{E}\left(\sum_{j=1}^{i}\left|x_{j}\right|\right)^{r-k},(k=0,1, \ldots, r), \\
& a_{k i}=-A_{j k}\left(z_{i}\right), \quad(k=1,2, \ldots, r-1), \\
& a_{0 i}=\sum_{k=1}^{r} \mathbf{E} z_{i}^{k} A_{r k}\left(z_{i}\right),
\end{aligned}
$$

let us assume that the expected values (16) exist for $k=1,2, \ldots, r-1$. Then the $r^{\text {th }}$ moment of the random variable $z_{n}$ exists and is equal to

$$
\mathbf{E} z_{n}^{r}=\sum_{k=0}^{r-1} \mathbf{E}\left(a_{k n} z_{n}^{k}\right) .
$$

The proof may be indicated in the following manner: Lemma 2 implies that the relation (18) (see the Russian text) holds. From the existence of the expected values (16) it follows that the $\mathbf{E}\left(a_{k n} z_{n}^{k}\right)$ exist. The relation (18) and the existence of the expected values $\mathbf{E}\left(a_{k n} z_{n}^{k}\right)$ imply that the assertion of the theorem is true.

The assertion of the theorem is also true, if the expected values (21) exist. The following theorem is a consequence of Theorem 5 :

Theorem 6. If the expected values (22) exist and the sequence $\left\{\mathbf{E}\left|x_{i}\right|^{r}\right\}$ is bounded, then the relation (17) holds. If $0<\varepsilon \leqq \mathbf{E}\left|x_{i}\right|$ for all $i$, then $\mathbf{E} n^{r}$ exists.

In the case of the existence of the expected values (23) the assertion of Theorem 6 is true, too.

Theorem \%. Let us suppose that the expected values (24) exist (r is a given positive integer $>1)$. If $0<\varepsilon \leqq \mathbf{E}\left|x_{i}-w_{i}\right|, \quad \mathbf{E}\left|x_{i}-w_{i}\right|^{r} \leqq \frac{1}{\varepsilon}(\varepsilon<1)$ for all $i$, there exists the expected value 


$$
\mathbf{E}\left(z_{n}-W_{n}\right)^{r}=\sum_{k=0}^{r-2} \mathbf{E}\left[b_{k n}\left(z_{n}-W_{n}\right)^{k}\right]
$$

where

$$
\begin{aligned}
W_{i} & =\sum_{j=1}^{i} w_{j}, \\
b_{k i} & =-A_{r k}\left(z_{i}-W_{i}\right), \\
b_{0 i} & =\sum_{k=1}^{r} \mathbf{E}\left(z_{i}-W_{i}\right)^{k} A_{r k}\left(z_{i}-W_{i}\right) .
\end{aligned}
$$

In the particular case $r=3$ it is sufficient to assume only the existence of the expected value (25).

Theorem 7 may be proved in a similar way as Theorem 5 .

Redakce: Matematický ústav Ceskoslovenské akademie věd, Praha IV, Loretánské n. 3, tel. 609-75. - Administrace: Nakladatelství Ceskoslovenské akademie věd, Praha II, Vodičkova 40, tel. 236375. - Vychází čtvrtletně - Roční předplatné Kčs 500,-, cena jednotlivého sešitu Kěs 125,- Novinové výplatné povoleno Okrskovým pošt. úřadem Praha 022: j. zn. 309-38-Ř-52.- Dohlédací poštovní úřad Praha 022.- Tiskne a expeduje Pražské tiskárny n. p., provozovna 05 (Prometheus), Praha VIII, třída Rudé armády 171 - Náklad 800 výtiskủ. 